Original Article

\title{
BASIC LIFE SUPPORT- A STITCH IN TIME
}

\author{
Muralee Mohan ${ }^{1}$, Rajendra Prasad', S.M. Sharma ${ }^{3}$, Deepthi Shetty ${ }^{4} \&$ Kalpa Pandya ${ }^{5}$ \\ ${ }^{1}$ Professor, ${ }^{2}$ Dean, Principal, ${ }^{3} \mathrm{HOD},{ }^{4}$ Assistant Professor, ${ }^{5}$ Post Graduate, Department of Oral \& M axillofacial Surgery, \\ A.B. Shetty Memorial Institute Dental Science, Nitte University, M angalore - 575 018, India. \\ Correspondence : \\ Kalpa Pandya \\ Post Graduate, Department of Oral \& M axillofacial Surgery, A.B. Shetty Memorial Institute Dental Science, \\ Nitte University, M angalore - 575 018, India. \\ Mobile: +9196321 65530 E-mail : kalpa.pandya@gmail.com
}

\begin{abstract}
:
Basic Life Support is the identification of emergency medical conditions such as stroke, cardiac arrest, foreign body obstruction; cardiopulmonary resuscitation (CPR) and defibrillation. The main aim of CPR is to increase the blood supply to the brain and the heart. Any delay in providing CPR reduces the chances of survival for the victim. Thus timely CPR is also mandatory. Training in Basic life Support skills is a must for medical and paramedical professionals and students. M oreover, it is essential to train even the layman in these skills as bystanders are the first and the best rescuers if trained adequately. American Heart Association (AHA) guidelines are one of the most widely accepted ones in terms of measures for basic life support. These guidelines are updated every five years. The last update was in 2010. Through, this article an attempt has been made to throw light on the 2005 protocol and 2010 updates as recommended by AHA.
\end{abstract}

Keywords: cardiac arrest, cardiopulmonary resuscitation, rescue breaths, chest compressions

\section{Introduction :}

Basic Life Support is a sequence of medical care which is provided as a life saving measure in cases of medical emergencies such as sudden cardiac arrest, drowning, chokingand trauma.

Sudden cardiac death [SCD] is thought to be a cause of one fourth of the human deaths. Of the various causes of sudden cardiac death, ventricular fibrillation is found to be the most common. [1]

The main aims of Basic Life Support include:

Dealing with the unconscious victim

Preventing further injury

Improving the chances of survival till definitive medical help arrives

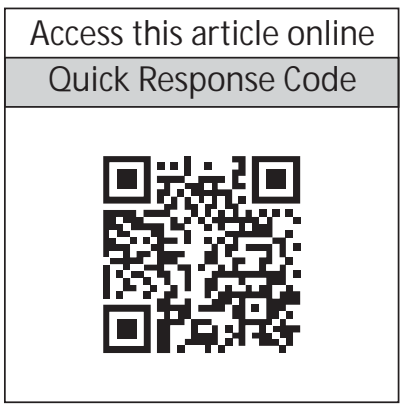

Considering the aims above, it becomes essential for even a layman to learn basic life support skills as bystanders are the best rescuers if trained efficiently.
The American Heart Association (AHA) recommends a four link Chain of Survival for resuscitation of a victim who has collapsed. [2]

It includes (Figure 1):

Activating the emergency medical services

Delivering Cardiopulmonary Resuscitation (CPR)

Shock delivery with a defibrillator

Post resuscitation care by healthcare organisations

\section{Checkfor response:}

The first step in the event of a collapse is to try to elicit a response from the adult. The rescuer may forcefully tap the patient and ask him if he is alright. If the patient responds, the rescuer must immediately call the nearest emergency medical help available. Ensuring this, he must return back to the victim and continue to monitor him.

\section{Call Emergency M edical Help:}

If the patient does not respond, the rescuer should immediately leave to call for emergency medical help. Care should to be taken to avoid panic and inform the emergency medical service of the exact venue of the occurrence. Following this the rescuer must return immediately and start with CPR. 
If two rescuers are available, one must start immediately with CPR while the other takes care of calling for emergency help. At no rate, should it be assumed that patient recovery will not require emergency help and the manoeuvres by the rescuers will be enough. Emergency help has to be called for without giving it a second thought or discussion. In case if the lone rescuer can conclude that the collapse is due to asphyxia of any cause, he must provide CPR (cycles) and then proceed onto contacting the emergency help.

\section{Openingthe Airway}

The manoeuvre indicated for this is a head tilt and a chin lift. However if any spine injury is suspected, a jaw thrust should be used instead of a head tilt. In the case, where jaw thrust is not possible, one must not hesitate to use a head tilt - chin lift procedure even in case of a suspected spine injury. [2]. This is important to consider as $2 \%$ individuals having blunt trauma have a chance of suffering an underlying spine injury. (3)

\section{Check for breathing}

One must Look-Listen-Feel for breathing. On failure to determine if any breathing is present, the rescuer must give 2 rescue breaths. [2]. We should not mistake occasional gasps as effective breaths. A few occasional gasps should be considered as no breathing and such a situation demands rescue breaths.

\section{Rescue Breaths}

As reported by Deakin et al only compression s have limited ability to maintain adequate gas exchange in cases of advanced stages of cardiac arrest. [1]

As per the AHA guidelines, a rescue breath must extend over a period of 1 second. The adequacy of the rescue breath is gauged on the basis of its ability to produce a noticeable rise of the chest. Very forceful breaths should be avoided as they can cause gastric inflation. It can cause aspiration, regurgitation, and restriction of lung movement and reduced respiratory compliance. [4]. Hence the need to extend one rescue breath, for a period of 1 second. The aim to notice a chest rise is to deliver air nearly equal to the tidal volume. The various modalities of delivering rescue breathsinclude,

1. Mouth-to-M outh Rescue Breathing

2. Mouth to barrier device

3. Mouth to nose

4. Bag mask ventilation

\section{Checkfor pulse}

It is often difficult to detect pulse in a collapsed victim even if it is present. In such cases the rescuer must not spend more than 10 seconds to detect pulse. After 10 seconds, failure of detection of pulse warrants start of CPR without wasting further time [2]. Carotid pulse is to be palpated. It is located by placing the fingers just between the trachea and the muscles at the side of the neck.

\section{Chest compressions}

It is essential to deliver effective chest compressions. Chest compressions are delivered in the region of the middle of the sternum between the nipple line. Compressions are delivered at the rate of 100 per minute with a compression depth of 1.5 to 2 inches. [2] Chest should be allowed to completely recoil after each compression. Any interruptions during the chest compressions should be reduced.

As per the AHA guidelines, with the victim in supine position, the rescuer kneels besides the victim's chest. The heels of the two palms are placed one over another with palms overlapped and parallel. The heel of the palm placed lower, is placed in the middle of the sternum on the nipple line.

A compression to ventilation ratio of 30:2 is recommended. In cases of cardiac arrest with an obstructed airway pulmonary ventilation assumes a great importance. Ventilation improves arterial oxygenation whereas in case of compressions only, a near desaturation of arterial oxygen concentration is seen. [5]

\section{Defibrillator}

As mentioned earlier ventricular fibrillation is most commonly found in the cases of sudden cardiac arrest. Defibrillation thus highly improves the chances of survival. 
An Automated External Defibrillator (AED) is commonly used for this purpose. These are devices which can identify if the victim requires shock. It can then deliver the required shock. CPR should be performed consistently until the AED arrives. Also if more than one rescuer is present, one should continue with CPR while the other attaches the AED pads.

The four universal steps for operating an AED as given by AHA are:

1. The AED is switched on.

2. AED pads attached to the bare chest of the victim.

3. Analyse the rhythm after "clearing" the victim. To "clear" the victim is to make sure that no one is touching the victim including the rescuer.

4. If the shock is required, command to clear the victim is given again by the AED.

5. Resume CPR after shock delivery or if shock is not required.

6. Shock is delivered after every 5 cycles or two minutes of CPR.

\section{0 updates in Basic Life Support as recommended by}

AHA:

\section{A-B-C to C-A-B}

The 2010 Guidelines for Cardiopulmonary resuscitation (CPR) and emergency cardiovascular care (ECC) [6] show a change in the sequence from Airway-BreathingCirculation to Circulation-Airway-Breathing (Figure 3). It was often seen that the chest compressions were given much later than actually recommended. This was due to the prolonged time sometimes required to open the airway or retrieve the ventilation device. When chest compressions are delivered first, the breaths should be given with a minimal delay.

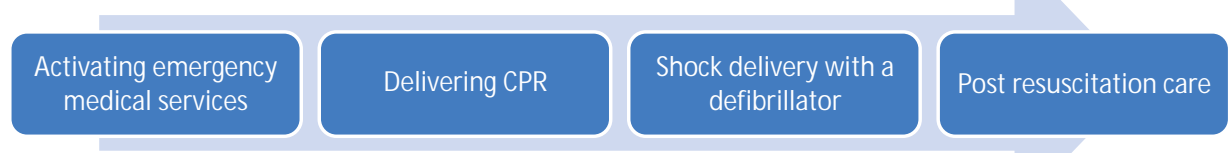

Figure 1

\section{Universal Algorithm}

Figure 2 demonstrates the algorithm for BLS as given in 2005 guidelines provided by AHA.

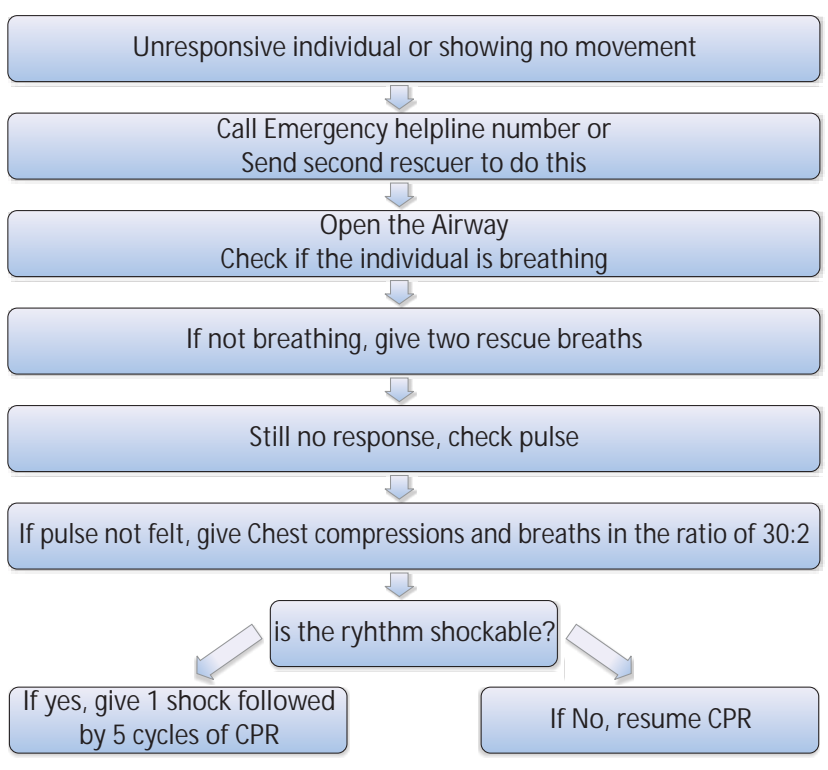

Figure 2

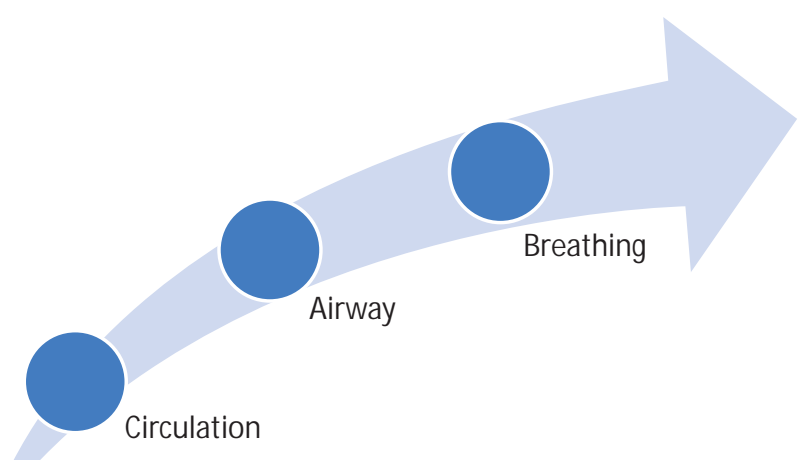

Figure 3

\section{CPR of High Quality}

To deliver CPR of high quality, 2010 AHA guidelines [6] recommend:

- A change of compression rate from approximately $100 /$ min to at least $100 / \mathrm{min}$.

- Compression depth recommended for adults is $5 \mathrm{~cm}$ or 2 inches. It is $4 \mathrm{~cm}$ or 1.5 inches in infants and $5 \mathrm{~cm}$ or 2 inches in children. This is approximately equal to one 
third the antero-posterior diameter in infants and children.

A very important recommendation includes a team approach rather than a single rescuer. This will allow the group of rescuers to perform different actions simultaneously making the process highly efficient.

\section{Eliminatinglook-listen-feel}

Rescuers often wasted time in checking if the victim is breathing. This often delayed the delivery of CPR. Also while looking for breathing, occasional gasps were often mistaken as breathing causing a failure of delivering CPR when the patient actually requiresit.

\section{References:}

1. Abhilash $\mathrm{SP}^{1}$, Namboodiri $\mathrm{N}^{2}$ Sudden cardiac death - Historical perspectives. Indian Heart J. 2014 January - February;66S1:S4-S9. doi: 10.1016/j.inj.2014.01.002. Epub 2014 Feb 11,

2. Part 4: Adult Basic Life Support Circulation. 2005;112:IV-19-IV-34; originally published online November 28, 2005

3. Hackl W, Hausberger K, Sailer R, Ulmer H, Gassner R. Prevalence of cervical spine injuries in patients with facial trauma. Oral Surg Oral Med Oral Pathol Oral Radiol Endod. 2001;92:370-376.

4. Guidelines 2000 for Cardiopulmonary Resuscitation and Emergency Cardiovascular Care. Part 3: adult basic life support. The American Heart Association in collaboration with the International Liaison Committee on Resuscitation. HYPERLINK "http://www.ncbi. nlm.nih.gov/pubmed/10966662" Io "Circulation."Circulation. 2000 Aug 22;102(8Suppl):122-59.

5. Dorph $E^{1}$ Wik L, Strømme TA, Eriksen M, Steen PA Resuscitation. Oxygen delivery and return of spontaneous circulation with ventilation:compression ratio 2:30 versus chest compressions only CPR in pigs 2004 $\mathrm{M}$ ar;60(3):309-18

6. TO - Field J M, Hazinski M F, Sayre M R, Chameides L,Schexnayder SM, Robin H Part 1: Executive Summary: 2010 American Heart Association Guidelines for Cardiopulmonary Resuscitation and Emergency Cardiovascular Care Circulation. 2010;122:S640-S656.

\section{Conclusion:}

The protocol for basic life support as given by AHA is being updated every five years on the basis of evidence based studies. Training and updating the knowledge in basic life support is mandatory for medical and paramedical professionals and students. Moreover, it is the duty of these personnel to enlighten the layman about the importance of learning skills of basic life support. A prompt action can prevent the medical condition to progress further and thus save a life. Indeed, a stitch in time saves nine. 